\title{
SiGe: A PROMISE INTO REALITY?
}

\author{
H.G. Grimmeiss, J. Olajos and J. Engvall
}

Department of Solid State Physics, University of Lund, Box 118, 22100 Lund, Sweden

The paper summarizes a few basic properties of SiGe showing that SiGe is an interesting material for high speed electronics. The advantage of using heterostructures in silicon-based technologies is demonstrated by taking SiGe heterobipolar transistors as an example. First results obtained with very fast and low-noise heterobipolar transistors are briefly mentioned. The paper is concluded by a short discussion of a few optoelectronic properties observed in various $\mathrm{Si} / \mathrm{Ge}$ and $\mathrm{Si} / \mathrm{Si}_{1-x} \mathrm{Ge}_{x}$ strained-layer superlattices and quantum wells with particular emphasis on electroluminescence properties.

PACS numbers: 71.55.Cn

\section{Introduction}

Information technology is one of the most important global key industries. The most important areas of information technology are probably microelectronics, software and data transfer. Restricting ourselves to microelectronics it is fair to say that the impressive progress in semiconductor electronics has only been possible due to an unique combination of basic conceptual advances, the perfection of new materials and the development of new device principles. Ever since the invention of the transistor, we have witnessed a fantastic growth in silicon technology, leading to more complex functions and higher densities of devices, such as the megabit memories. This development would hardly be possible without an increasing understanding of semiconductor materials and new concepts in material growth techniques which again and again made it possible to fabricate previously not known semiconductor structures.

Microelectronics means the multifunctional integration of electronic devices. Since the beginning of this important field the goals for the development of microelectronics have always been to further increase the packing density (i.e. the number of devices per unit area), the integration level (i.e. the number of functions on a chip), the signal processing rate (i.e. the information transfer per unit time), and the multifunctionality (i.e. the variety of devices). These goals are accompanied by requirements of the market which primarily imply to be most inexpensive. Costs are easiest minimized by increasing the production yield and having the most effective process technology. How important it is to be first on the market is still discussed and the answer certainly depends on the product and the company. The question now arises, how these goals can be accomplished. Various attempts have been made and with respect to semiconductor materials three concepts are of particular interest to be mentioned briefly: 
(1) III-V semiconductor compounds are known to have very interesting properties for applications in microelectronics and have successfully been used in fabricating fast circuits of high packing density. They form the basic materials for the growth of different kinds of heterostructures which for a long time have been the main reason for the steady interest in these compounds. Heterostructures are the basis of very fast devices such as the high electron mobility transistor, and important applications in optoelectronics such as the lasers. In spite of these unique properties, the market share of III-V devices has always been less than $10 \%$ world-wide. Production costs and the absence of native oxides are often mentioned as the main factors contributing to the small market share.

(2) Three-dimensional, stacked semiconductor structures are another concept which is often tried and which certainly will acquire further interest in the future. Considering how difficult it is to keep high yields already with two-dimensional circuits it is easily realized that these difficulties certainly are still more severe for stacked structures which is one of the reasons for the limited applicability of this concept so far.

(3) The reduction of the structure size within silicon technology is often considered as the main concept in microelectronics. Moore's law has been valid since the very beginning of microelectronics and there are good reasons to believe that it will, at least in principle, continue to be valid for quite a while. Though silicon devices have a market share of more than $90 \%$ world-wide due to low costs and high yields, they have so far proven to be rather inefficient in optoelectronic applications. Since it is difficult to grow heterostructures using only silicon, even the most efficient silicon technology will never be able to compete with III-V semiconductor compounds at least not with respect to the signal processing rate and light emitting properties.

Recently, a new technology has been developed by using both silicon and germanium either as elements or as alloys. This technology which already has shown to be of great interest for microelectronics and, hence, for information technology in general, makes it possible to grow similar heterostructures as with III-V compounds and is therefore in principle able to combine the advantages of the silicon technology with those of the III-V or other semiconductor compounds. Obviously, this technology is not limited to silicon and germanium but may also include other group IV elements. First results obtained in this respect are very encouraging [1].

The purpose of this paper is to summarize a few basic properties of SiGe which may be useful for a better understanding why SiGe is an interesting material for microelectronics. Heterobipolar transistors (HBT) will be used as an example to demonstrate the advantage of using heterostructures in silicon-based technologies for the fabrication of very fast and low-noise transistors with low power consumption. The paper is concluded by presenting a few results which recently have been obtained regarding the optical properties of SiGe low dimensional structures.

\section{Heterobipolar transistors}

Ge has a lattice constant which is about $4 \%$ larger than the one of silicon. This difference in the lattice constant has for a long time been considered as a severe obstacle for growing heterostructures. First attempts have indeed not been 
very successful and first the pioneering work with III-V semiconductor compounds initiated in the middle of the ' 80 a break-through in the silicon based heterostructure device work.

As a consequence of the lattice mismatch, the unit cell of SiGe alloys is larger than the one of silicon and SiGe epitaxial layers grown on a silicon substrate are therefore strongly compressed. The thickness of pseudomorphic (commensurate or strained) epitaxial layers is therefore characterized by a critical thickness which depends on composition. The critical thickness of pseudomorphic $\mathrm{Si}_{1-x} \mathrm{Ge}_{x}$ layers of $x=0.20$, for example, results in a value of slightly less than $100 \mathrm{~nm}$.

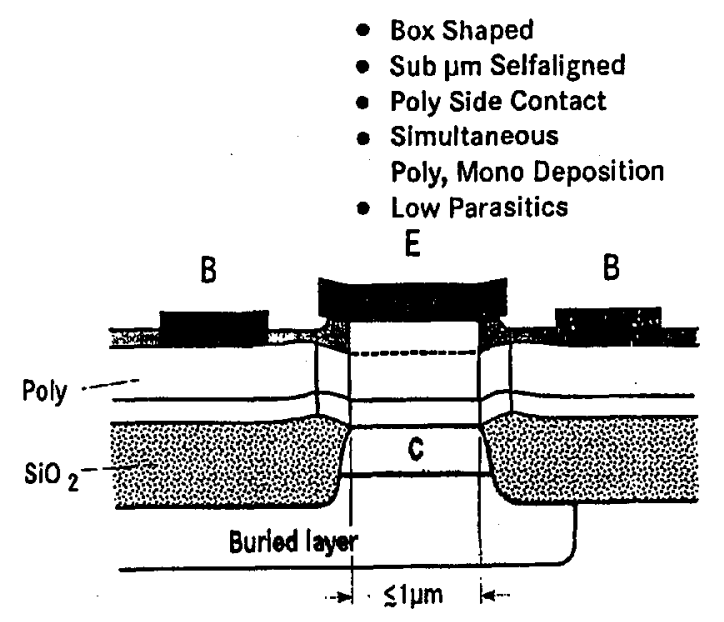

Fig. 1. Differential SiGe HBT, suggested by the Daimler-Benz.

Figure 1 shows a box shaped, differential SiGe HBT suggested by the Daimler-Benz research laboratory in Ulm. It is a sub $\mathrm{mm}$ self-aligned structure with poly silicon and SiGe side contacts simultaneously deposited with the monolayers. The emitter (E) and collector (C) are doped silicon whereas the base (B) is a $\mathrm{Si}_{1-x} \mathrm{Ge}_{x}$ alloy with $x$ around $20 \%$.

The band structure of such an HBT is exhibited in Fig. 2. It is important to realize that the difference in the band-gap energy $\Delta E_{\mathrm{g}}$ between the emitter and base is almost completely absorbed by a lowering of the conduction band edge. Compared with a silicon bipolar transistor (BT), this implies that electrons have to overcome a much lower energy barrier when being injected from the emitter. into the base whereas holes face the same energy barrier in both cases when diffusing from the base to the emitter. Considering that the intrinsic free carrier concentration in $\mathrm{SiGe}, n_{\mathrm{i}}(\mathrm{SiGe})$ is given by

$$
n_{\mathrm{i}}(\mathrm{SiGe})=\sqrt{N_{\mathrm{C}} N_{\mathrm{C}}} \exp \left(\frac{E_{\mathrm{g}}(\mathrm{SiGe})}{2 k T}\right) \gg n_{\mathrm{i}}
$$

and, hence, is considerably higher than in $\mathrm{Si}$, it is quite obvious that the electron 


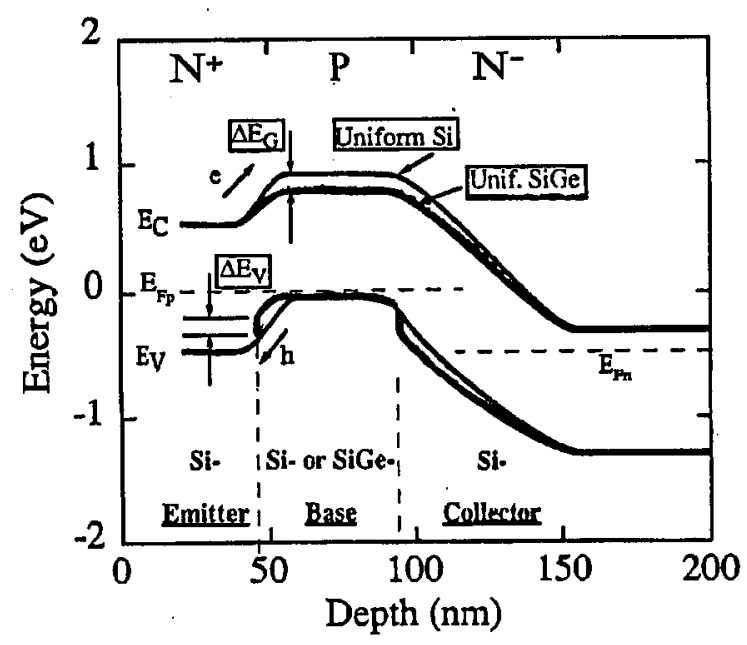

Fig. 2. Band-diagram of an HBT.

current across the base

$$
j_{n} \sim \frac{q D_{n} n_{\mathrm{i}}^{2}(\mathrm{SiGe})}{N_{\mathrm{B}} w_{\mathrm{B}}} \exp \left(\frac{q V_{\mathrm{EB}}(\mathrm{SiGe})}{2 k T}\right)
$$

is much larger in an HBT than in a silicon transistor whereas the hole current across the emitter is the same in both cases.

For a common-emitter configuration the current gain can therefore be approximated by

$$
\beta=\frac{j_{n}}{j_{p}} \sim \frac{D_{n} N_{\mathrm{E}} w_{\mathrm{E}}}{D_{n} N_{\mathrm{B}} w_{\mathrm{B}}} \frac{n_{\mathrm{i}}^{2}(\mathrm{SiGe})}{n_{\mathrm{i}}^{2}(\mathrm{Si})}
$$

Since $n_{\mathrm{i}}^{2}(\mathrm{SiGe}) / n_{\mathrm{i}}^{2}(\mathrm{Si})$ is proportional to $\exp \left\{\left[E_{\mathrm{g}}(\mathrm{Si})-E_{\mathrm{g}}(\mathrm{SiGe})\right] / k T\right\}=$ $\exp \left(\Delta E_{\mathrm{g}} / k T\right)$ it is readily seen that the current gain in an HBT, without changing the geometry or doping, is, compared with a silicon transistor, a factor of about $\exp \left(\Delta E_{\mathrm{g}} / k T\right)$ larger. Taking $x=0.20$, we obtain $\Delta E_{\mathrm{g}} \approx 160 \mathrm{meV}$ implying that the current gain is increased by a factor of about 600 in an HBT by no further adjustments than by replacing the base layer by $\mathrm{Si}_{0.80} \mathrm{Ge}_{0.20}$. For obvious reasons, the emitter in a silicon bipolar transistor is always much higher doped than the base. By doping the base of an IIBT with $x=0.30$ four times higher than the emitter and decreasing the base width to about $30 \mathrm{~nm}$, a current gain of 4000 has been achieved at room temperature which increased to about 10000 at $120 \mathrm{~K}$. Higher doping of the base than the emitter is allowed in an HBT without a negative effect on the current gain since holes have to overcome a considerable larger potential barrier than electrons. A higher base doping also tolerates a decrease in the base width without increasing the base resistance or loosing current gain. This is easily shown by comparing an HBT with a silicon transistor of similar current gain.

$$
\beta_{\mathrm{Si}}=\beta_{\mathrm{SiGe}}=1 \approx \frac{N_{\mathrm{B}(\mathrm{Si})} w_{\mathrm{B}(\mathrm{Si})}}{N_{\mathrm{B}(\mathrm{SiGe})} w_{\mathrm{B}(\mathrm{SiGe})}} \frac{n_{\mathrm{i}}^{2}(\mathrm{SiGe})}{n_{\mathrm{i}}^{2}(\mathrm{Si})}=\frac{N_{\mathrm{B}(\mathrm{Si})} w_{\mathrm{B}(\mathrm{Si})}}{N_{\mathrm{B}(\mathrm{SiGe})} w_{\mathrm{B}(\mathrm{SiGe})}} 600
$$


assuming similar diffusion coefficients $D$ in $\mathrm{Si}$ and $\mathrm{Si}_{0.80} \mathrm{Ge}_{0.20}$ and similar dopings and geometries of the emitter in both cases. Equation 4 readily shows that if the base doping of the HBT is increased by a factor of about 1800 , the base width can be reduced by a factor of 3 without losing current gain. Whereas current gain is not of major concern in microelectronics, the signal processing rate certainly is. This rate depends critically on the base width as shown by the following simplified considerations.

The frequency $f_{T}$ at which the magnitude of the incremental short-circuit common-emitter current gain extrapolates to unity is given by

$$
f_{T}=\frac{1}{2 \pi \tau_{\mathrm{EC}}}
$$

where $\tau_{\mathrm{EC}}$ is the transit time of the carrier between emitter and collector and given by the sum of the emitter, base and collector transit times and the emitter and collector charge times. The base transit time without built-in-field can be approximated by

$$
\tau_{\mathrm{B}} \approx \frac{w_{\mathrm{B}}^{2}}{2 D_{n}}
$$

and gives in general the largest contribution to $\tau_{\mathrm{EC}}$. Hence, increasing the doping level of the base and decreasing the base width $w_{\mathrm{B}}$ by a factor of about 3 may increase $f_{T}$ by almost an order of magnitude. $f_{T}$ values of SiGe HBTs above $100 \mathrm{GHz}$ have already been achieved.

Though $f_{T}$ is an interesting transistor parameter, it is worthwhile to mention that the frequency $f_{\max }$ at which power amplification is 1 , is in many respects a still more important parameter. $f_{\max }$ is given by

$$
f_{\max }=0.2 \sqrt{\frac{f_{T}}{R_{\mathrm{B}} C_{\mathrm{BC}}}}
$$

where $R_{\mathrm{B}}$ is the base resistance (including connections and contacts) and $C_{\mathrm{BC}}$ is the collector-base capacitance. Assuming similar $f_{T}$ (i.e. $w_{\mathrm{B}}$ ) and $C_{\mathrm{BC}}$ as well as similar emitter properties and current gain $\left(b_{\mathrm{SiGe}}=b_{\mathrm{Si}}\right)$ for an HBT and silicon BT we obtain from Eqs. (5) and (7) the ratio of the maximum oscillator frequency $f_{\max }$ by considering $R_{\mathrm{B}} \sim 1 / N_{\mathrm{B}}$

$$
\frac{f_{\max }(\mathrm{SiGe})}{f_{\max }(\mathrm{Si})} \sim \sqrt{\frac{N_{\mathrm{B}}(\mathrm{SiGe})}{N_{\mathrm{B}}(\mathrm{Si})}} \sim \frac{n_{\mathrm{i}}(\mathrm{SiGe})}{n_{\mathrm{i}}(\mathrm{Si})} .
$$

For $x=0.20$, this ratio is about 20 . Although a factor of 20 is difficult to obtain with the above assumptions, since $f_{T}$ is not only dependent on $I_{\mathrm{B}}$ and, hence, $w_{\mathrm{B}}$, Eq. (8) nevertheless shows that considerably higher $f_{\max }$ values are achievable in SiGe HBT than in silicon bipolar transistors. Without going into details it is also worth mentioning that considerable lower noise figures have been reported at $10 \mathrm{GHz}$ for Si/SiGe IIBT than for Si BT as well as AlGaAs/GaAs and InGaAs/InP HBT.

\section{SiGe optoelectronics}

HBTs are only one example how useful SiGe heterostructures can be for microelectronics. Recently, interesting results have been obtained with regard to 
optical properties of various $\mathrm{Si} / \mathrm{Ge}$ and $\mathrm{Si} / \mathrm{Si}_{1-x} \mathrm{Ge}_{x}$ strained-layer superlattices (SLSs) and quantum wells (QWs). The growth of lattice mismatched materials results either in a strained-layer configuration, if the grown layer is thinner than the critical thickness, or in a strain relaxation with simultaneous formation of misfit dislocations. Consequently, the realization of $\mathrm{Si} / \mathrm{Ge}$ based structures for optical or electrical applications must involve either a very thin epitaxial layer which does not exceed the critical thickness, or if a thicker layer is needed, a method which during growth reduces the density of threading dislocations extending into the active region. Additional difficulties arise with regard to intermixing of the two atomic species into each other when truly short-period structures are considered.

In this section we will discuss some of the recently obtained results which have improved our understanding of optical and electro-optical properties of $\mathrm{Si} / \mathrm{Ge}$ SLSs based on the two different approaches.

\subsection{Strain-symmetrized $S i_{m} G e_{n}$ superlattices}

One of the driving forces to the rather extensive experimental [2-6] and theoretical [7-13] work performed on these structures is the suggestion, based on rather simple "Brillouin-zone folding" arguments, that a "quasi-direct" semiconductor material should be obtained from originally indirect semiconductors if they were grown in a special way, involving alternating layers of the two different species [7]. In the case of $\mathrm{Si} / \mathrm{Ge}$, the basic idea is to grow a superlattice with a period of 10 monolayers (MLs). For this periodicity it is assumed that the conduction-band (CB) minima, which are located at 0.85 in the $\Gamma-X$ direction for bulk $\mathrm{Si}$, are folded back to the $G$ point. In this simple model, an enhanced oscillator strength is expected for the so-called "quasi-direct" transitions between the valence band and the twofold degenerate conduction band edge. More elaborate band structure calculations [ $8,11-13]$ have indeed predicted an enhanced oscillator strength for these transitions. The resulting band structure of a short-period, strained-layer superlattice will, however, be determined not only by zone-folding and confinement but also by the actual strain situation. In the case of Si/Ge SLSs, grown lattice-matched to $\mathrm{Si}[001]$-substrates, it has been found that the in-plane $(x, y)$ fourfold conduction band minimum is lowest in energy. No zone-folding is expected for these minima. In order to bring the twofold $(z)$-CB-minima below the fourfold in-plane CB-minima, deformation-potential theory together with an effective-mass (EM) calculation suggests that the Si-layers in the SLS must be under a lateral tensile strain. This can be accomplished if the SLS is grown on a substrate with a lattice constant between that of $\mathrm{Si}$ and Ge. A substrate material other than $\mathrm{Si}$ in Si-based microelectronics is for obvious reasons not very appealing. Strain-adjustment on a Si-substrate can, however, be achieved by the concept of "virtual-substrates" [14] which involves a partially relaxed $\mathrm{Si}_{1-x} \mathrm{Ge}_{x}$ alloy buffer layer. The Ge concentration in the buffer is then chosen such that the resulting lattice constant of the alloy provides a strain distribution for the SLS which is grown on top of the buffer which guarantees that the lateral strains in the Si- and Ge-layers are of equal magnitude but of opposite sign. In this way, the integral of the built-in elastic energy in the SLS is zero and no further relaxation occurs, even for very thick superlattices. The relaxed $\mathrm{Si}_{1-x} \mathrm{Ge}_{x}$ alloy buffer layer, 
however, contains a high density of dislocations which extend into the SLS region and reduces the performance of the superlattice. During the last years it was shown that thick buffers with a stepwise or linearly increasing Ge concentration have an improved crystalline quality and an almost 4 orders of magnitude lower threading dislocation density than thin buffer layers.

In the next sections we will discuss the optical properties of a set of SLSs which were grown under such optimized conditions.

The samples described in this paper were prepared in a Si-MBE chamber at the Daimler-Benz Research Center in Ulm and comprised both undoped $\mathrm{Si}_{m} \mathrm{Ge}_{n}$ SLSs as well as superlattices grown in the depletion-region of $p-n$ junctions. In the case of undoped SLSs, a series was prepared with $m / n$ ratios of nominally $9 / 6$, $6 / 4$, and $3 / 2$, respectively. The buffer layer consisted of a step graded alloy buffer $6500 \AA$ thick, followed by a $5000 \AA$ thick $\mathrm{Si}_{0.6} \mathrm{Ge}_{0.4}$ alloy.

\subsection{Photoluminescence}

Figure 3 shows PL spectra which have been obtained from a study of three SLS samples, namely $(\mathrm{Si})_{9} /(\mathrm{Ge})_{6},(\mathrm{Si})_{6} /(\mathrm{Ge})_{4},(\mathrm{Si})_{3} /(\mathrm{Ge})_{2}$ and a reference $\mathrm{Si}_{0.6} \mathrm{Ge}_{0.4}$ alloy sample grown under similar conditions as the superlattices.

The dominant luminescence signal of each sample consisted of a no-phonon (NP) peak and a transverse-optical (TO) phonon replica. The spectra have been corrected with regard to the response of the spectroscopic system. It is interesting to note that the superlattice with the 10 -monolayer periodicity, i.e. $(\mathrm{Si})_{6} /(\mathrm{Ge})_{4}$, exhibits the strongest photoluminescence (PL) for the NP line as compared with

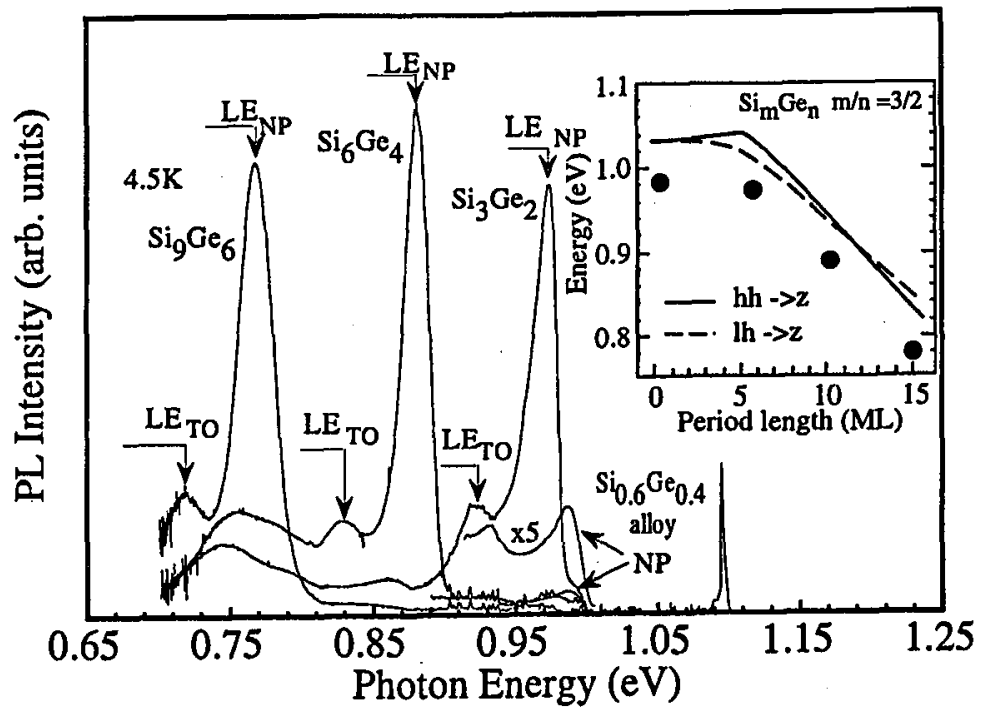

Fig. 3. PL spectra for $\mathrm{Si}_{9} \mathrm{Ge}_{6}, \mathrm{Si}_{6} \mathrm{Ge}_{4}, \mathrm{Si}_{3} \mathrm{Ge}_{2}$, and a $\mathrm{Si}_{0.6} \mathrm{Ge}_{0.4}$ alloy. 
the TO replica. The PL properties of these samples have been discussed in detail in an earlier communication [6]. It has been shown that the luminescence is consistent with a model involving near-band-gap recombinations over excitons localized to potential fluctuations caused by variations in the strain and Ge concentration. Taking into account the fact that the thickness of the SLS region is much smaller than the one of the $\mathrm{Si}_{0.6} \mathrm{Ge}_{0.4}$ alloy layer, the intensity of the $\mathrm{LE}_{\mathrm{NP}}$ (LE = localized exciton) line is enhanced by about a factor of 150 . At lower energies, defect-related features are observed in all three samples. The inset of Fig. 3 exhibits the energy gaps between the hh and $\mathrm{lh}$ valence band states and the twofold degenerate $(z)$ conduction band states for strain-symmetrized $\mathrm{Si}_{m} \mathrm{Ge}_{n}$ SLSs with a nominal value of $m / n=1.5$ as calculated with the EM (effective mass) approach. The systematic shift of the band-gap PL (photoluminescence) with increasing period length for the $\mathrm{Si}_{9} \mathrm{Ge}_{6}$ and $\mathrm{Si}_{6} \mathrm{Ge}_{4}$ samples is well described by these calculations. Similar systematic shifts of the onset of interband transitions on both undoped superlattices [15] as well as diode samples [5] have been studied earlier. Since the PL is observed very close in energy to these onsets the luminescence is best understood as (near-) band-gap luminescence. To further strengthen our assumption that the PL originates from interband transitions and to learn more about the physics behind the observed spectra, additional experiments involving hydrostatic pressure were performed.

The application of external hydrostatic pressure to a semiconductor results in a change of the lattice constant and therefore in the bond lengths which influences the band structure and electrical properties of the solid. These changes may give interesting information also on the optical properties of the samples. The energy shift of a luminescence line, for example, occurs as a function of applied hydrostatic pressure in different ways depending on whether it originates from a (near-) band-gap transition or a recombination over a deep defect level. A detailed study of the photoluminescence spectra of these samples under applied hydrostatic pressure has been performed recently [16]. Figure 4 shows an example of such a measurement obtained with a nominal $\mathrm{Si}_{6} \mathrm{Ge}_{4}$ SLS sample. Clearly, the NP lines shifted linearly to lower energies without any significant change in the amplitude.

The PL spectra exhibited, however, in addition to the NP lines other somewhat broader features which at atmospheric pressure were weakly observed at lower energies. These lines gained in intensity with increasing pressure and showed a positive pressure coefficient suggesting that other recombination processes, i.e. non-interband transitions are involved in these features. Though the chemical origin of the defect-related transitions is at present still unknown, these experiments made it possible to show for the first time experimentally that both defect-related $\mathrm{PL}$ as well as band-gap luminescence are observed in short-period $\mathrm{Si} / \mathrm{Ge}$ superlattice systems. The stress dependence was modelled with the methods described in the previous section. Although an externally induced, pure hydrostatic strain does not split the conduction- and valence-band edges a shift of the weighted averages is nevertheless introduced. The splittings from the weighted averages due to the built-in biaxial compression (Ge-layers) and tension (Si-layers) as well as the slightly non-hydrostatic part of the external pressure have to be calculated for the valence-band multiplet at $\Gamma_{25^{\prime}}$ and for the conduction-bands at both $L$ and $\Delta_{1}$ in 


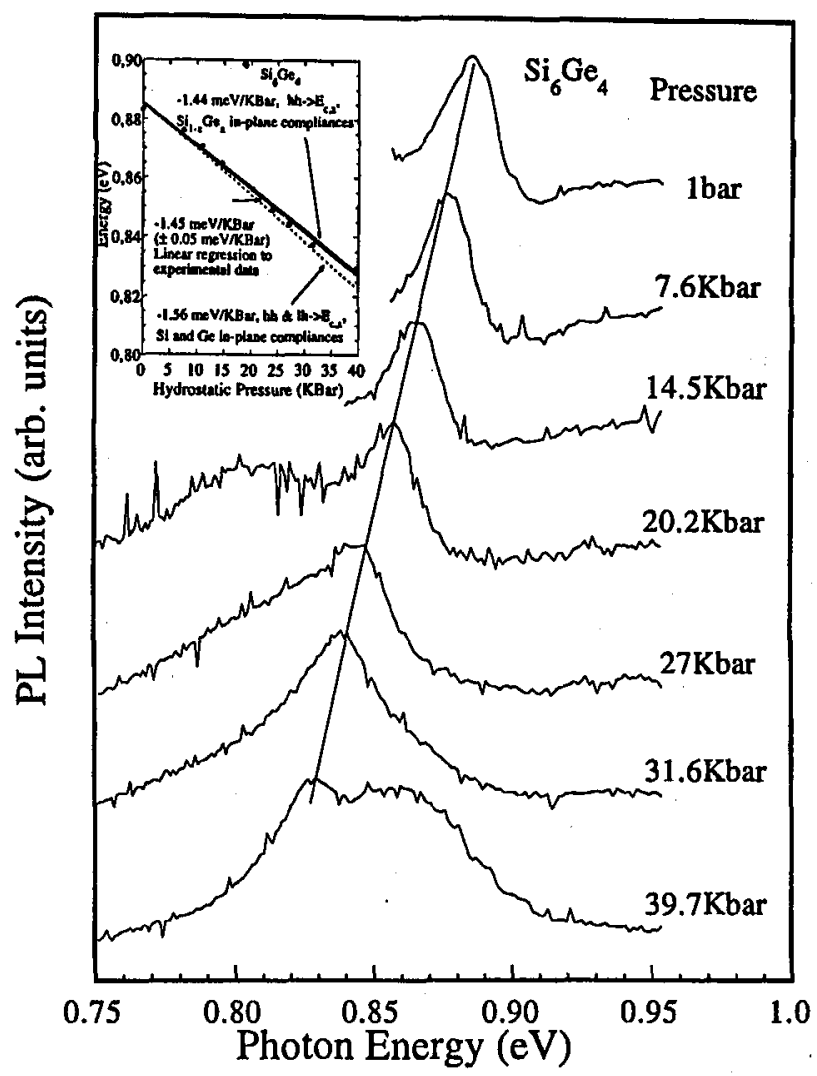

Fig. 4. PL spectra as a function of applied hydrostatic pressure. In the inset a comparison with various models is shown.

the Brillouin zone. The hydrostatic influence is then introduced as a relative shift of the valence- and conduction-band weighted averages since the absolute quantities are very difficult to determine experimentally as well as theoretically. The differences in band-offsets due to the extra hydrostatic pressure is then included around the weighted averages of the valence- and conduction bands.

The calculated pressure dependencies for the $\mathrm{Si}_{6} \mathrm{Ge}_{4}$ superlattice and the experimentally observed energy shifts of the NP lines are compared in the inset of Fig. 4. It is readily seen that the parameter-free theoretical slopes obtained from an approach where the $\mathrm{Si}$ and Ge layers in the SLS are treated as having the in-plane compliance of the thick $\mathrm{Si}_{1-x} \mathrm{Ge}_{x}$ alloy buffer (solid lines) coincide very well with a linear regression of the experimental data (dots). For comparison, the data from the model where the $\mathrm{Si}$ and Ge layers are treated as free-standing layers are also shown (dashed lines). In this case the agreement between theory and experiment is less favourable. 


\subsection{Electroluminescence}

Recently, enhanced electroluminescence (EL) has been observed at room temperature from $\mathrm{Si}_{6} \mathrm{Ge}_{4}$ strain-adjusted superlattice $p^{+} n$ junction in the region 1.3-1.7 $\mathrm{mm}(0.70-0.95 \mathrm{eV})[17]$. Absorbance, capacitance-voltage $(C-U)$, intensity versus current $(L-I)$, and voltage-intensity $(U-L)$ measurements suggested that the observed EL originates from transitions over a defect-related recombination center $(0.77 \mathrm{eV})$ as well as from band-to-band recombination processes $(0.88 \mathrm{eV})$ in the SLS. The quantum efficiency of the band-gap electroluminescence is, however, strongly dependent on the defect- and dislocation density in the active regions. The defect concentration is intimately coupled to the buffer-layer quality and design as discussed in the previous section. How efficient the infrared light is coupled out from the LED depends on what type of ridge waveguide structure is used. Figure 5 shows room-temperature electroluminescence spectra of samples based

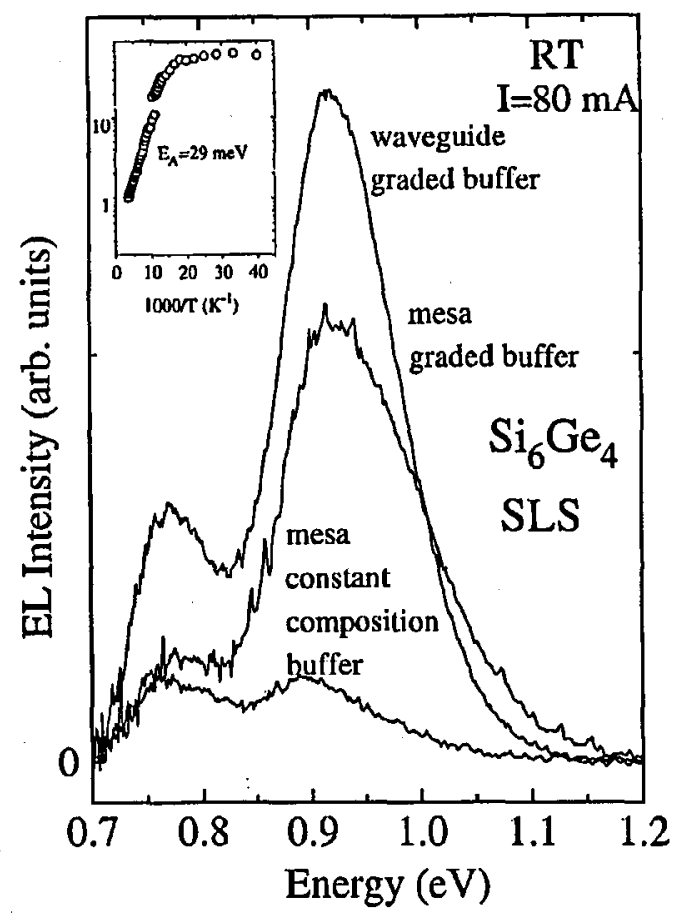

Fig. 5. A comparison of the EL intensity from a $\mathrm{Si} / \mathrm{Ge} \mathrm{SLS}$ grown under different conditions.

on different buffer layer designs. A considerable increase in the band-gap emission has been observed for mesa-diodes grown on a step-graded buffer at normal incidence at about $0.9 \mathrm{eV}$ due to a lower concentration of non-radiative recombination channels. Further increase in the light output has been obtained by overgrowing the superlattice with a thick $\mathrm{Si}_{0.6} \mathrm{Ge}_{0.4}$ alloy waveguide layer and structuring it into a waveguide edge emitter. Regardless whether the samples are grown with a 
waveguide layer on top or not, one would expect a strong polarization dependence of the optical properties because the strain in the SLS splits the valence bands, with the heavy-holes being lower in energy for several period-lengths. A waveguide geometry may also result in a different type of asymmetry since different coupling losses are expected for different polarizations of the light. This uncertainty raises therefore the question to what degree polarization originates from the electronic structure or from the geometry. In a photocurrent measurement, the light may still contribute to the photocurrent signal after many internal scattering phenomena. It is therefore possible that the incident light, completely polarized in one direction, changes polarization upon scattering and is subsequently absorbed.

This implies that the difference in the photocurrent signal between two polarizations is reduced and, hence, that the difference observed in a transmission measurement has to be greater than the one observed in a photocurrent measurement.

We have studied the polarization dependence of two different samples with graded buffers (B2710 and B2805). Figure 6 shows the short circuit current $\left(I_{\mathrm{SC}}\right)$ spectra of sample B2805 with a waveguide for two different polarizations. The mesa spectrum has been expanded to coincide with the waveguide spectra. The spectral dependence close to the onset is similar for both mesa and waveguide.

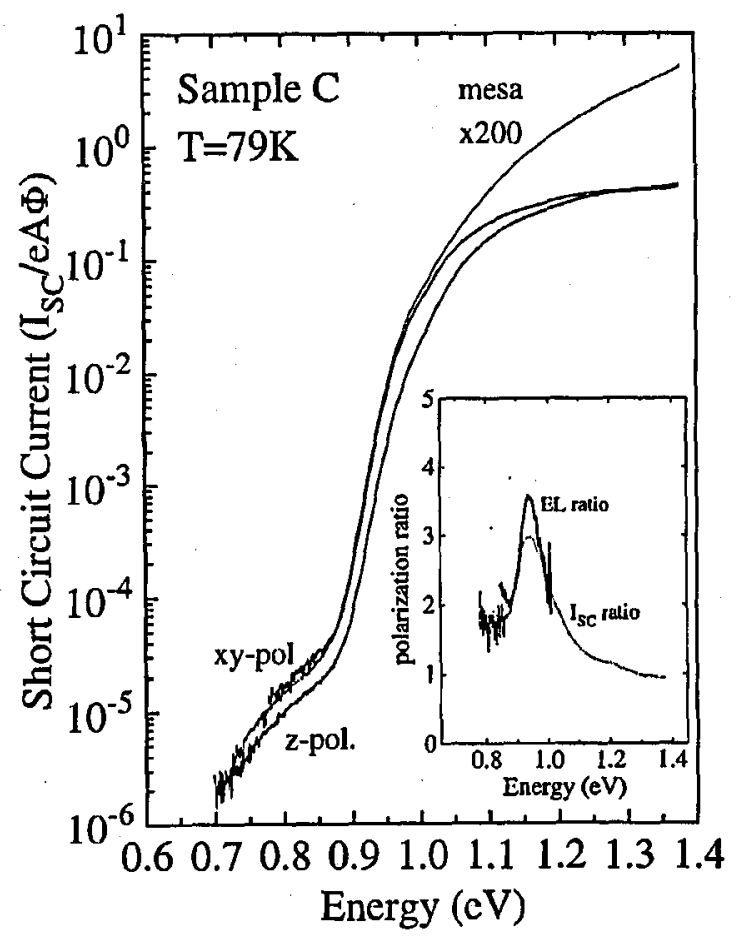

Fig. 6. $I_{\mathrm{SC}}$ spectra from a $\mathrm{Si}_{6} \mathrm{Ge}_{4}$ in different polarizations. 
The spectra of the waveguide sample deviates from the spectrum of the mesa at higher photon energies. Since the efficiency of the waveguide is close to unity, this implies that the signal is no longer proportional to the absorption coefficient, while only a few percent of the light is absorbed in the mesa diode. In both samples a difference in the signal amplitude has been observed for different polarizations.

The $X Y$-polarization ( $E$-field parallel to epilayer) results in a larger signal than the $Z$-polarization ( $E$-field perpendicular to epilayer). The inset in Fig. 6 shows the polarization dependence by exhibiting the $I_{\mathrm{SC}}$ for the polarization $X Y$ divided by the $I_{\mathrm{SC}}$ for the $Z$-polarization obtained in two samples with different thickness of the waveguide.

The fact that this ratio is one for high photon energies is not surprising since a ratio of one is expected if all the light is absorbed for both polarizations. It is also seen that the ratio is close to one below the interband onset i.e. in a region where the $I_{\mathrm{SC}}$ is caused by defect assisted absorption processes. However, for energies between 0.9 and $1 \mathrm{eV}$ a strong increase in the absorbance for the in-plane polarization is observed as compared with the perpendicular polarization. The EL observed in the waveguide samples exhibited also a polarization dependence. It is readily seen in the previous inset that the same anisotropy as detected for the absorption is also seen for the luminescence.

\section{Thin Ge layers embedded in Si}

Another approach to increase the oscillator strength in Si/Ge microstructures is based on the observation that an originally forbidden zero-phonon transition in indirect bulk semiconductors can be changed to an allowed transition if there is some mixing of the bulk momenta. This occurs, for example, when an electron is scattered at an Si-Ge interface [10]. One could therefore expect to initiate such transitions simply by growing one or more thin Ge layers within a thick Si matrix. Since it is mainly the $\mathrm{Si}-\mathrm{Ge}$ interface which is of importance, the total thickness of the Ge-layer can be kept low, i.e. 2-3 ML, implying that the critical thickness does not have to be exceeded. In addition, strong confinement for holes is predicted for such structures.

Layer sequences based on this concept were grown at the Daimler-Benz Research Center consisting of a series of $\mathrm{Si}_{17} \mathrm{Ge}_{2}$ structures repeated 2, 5, and 10 times in the depletion region of $p-i-n$ diodes. The electroluminescence spectra of this series taken at $56 \mathrm{~K}$ with an injection current density of $20 \mathrm{~A} / \mathrm{cm}^{2}$ is shown in Fig. 7. A strong EL emission which consists of a doublet peak at around $1.0 \mathrm{eV}$ is observed for all samples. The peak position is in good agreement with the onset of interband transitions in the SLSs and it is therefore believed that the luminescence originates from a (near-) band-gap emission.

The inset of Fig. 7 shows a comparison between the EL spectrum of the sample with $N=2$ periods and the $\mathrm{PL}$ from an ordinary pseudomorphic $\mathrm{Si}_{1-x} \mathrm{Ge}_{x}$ alloy quantum well. PL properties of such structures have been extensively studied over the last years and interpreted as consisting of a no-phonon line and a set of phonon replicas reflecting the various possible combinations of $\mathrm{Si}-\mathrm{Si}, \mathrm{Si}-\mathrm{Ge}$, and $\mathrm{Ge}-\mathrm{Ge}$ vibrations in a $\mathrm{Si}_{1-x} \mathrm{Ge}_{x}$ alloy with fairly low Ge concentration. The inset of Fig. 7 clearly shows that the energy difference between the two peaks in 


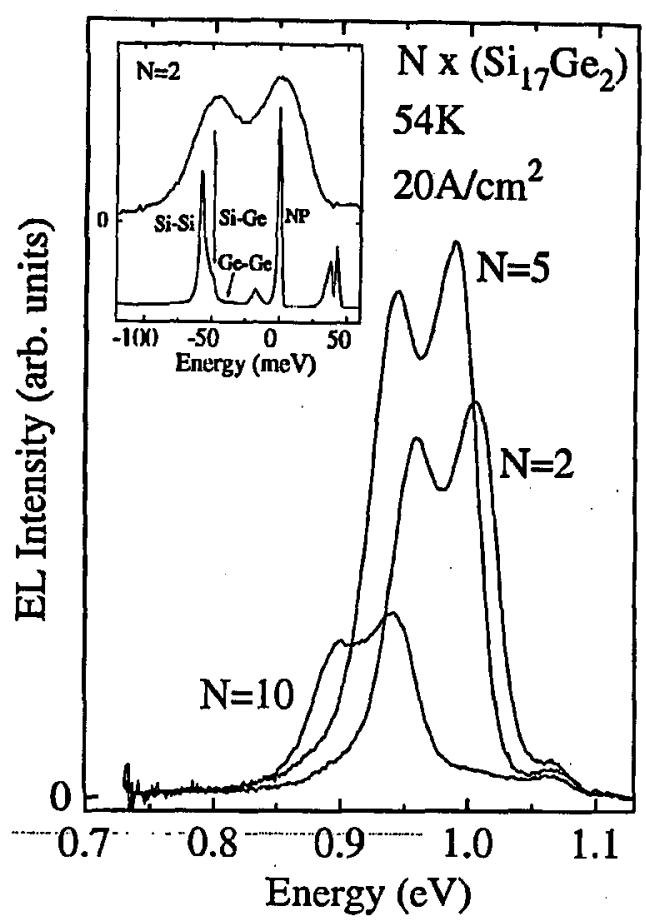

Fig. 7. EL spectra for three Si/Ge MQWs. In the inset a comparison between the EL from these samples and a $\mathrm{PL}$ spectra from a $\mathrm{Si}_{1-x} \mathrm{Ge}_{x}$ alloy $\mathrm{QW}$ is shown.

the EL spectrum of the $\mathrm{Si}_{17} \mathrm{Ge}_{2}$ SLS corresponds to the difference between the no-phonon line and the TO-replica of the $\mathrm{Si}-\mathrm{Ge}$ vibration in the alloy quantum well. It is therefore possible to attribute the $\mathrm{EL}$ spectrum of the $\mathrm{Si}_{17} \mathrm{Ge}_{2}$ structure to a no-phonon line and a TO phonon-replica due to $\mathrm{Si}-\mathrm{Ge}$ vibrations from atoms at the interface of the Ge-layers. It is worth noting that only the $\mathrm{Si}-\mathrm{Ge}$ mode is observed which is essentially the only bond present in such a structure. This gives further strength to the theoretical prediction of the importance of the $\mathrm{Si}-\mathrm{Ge}$ interface. With increasing number of periods $N$, the EL signal shifts to lower energies and decreases in intensity. The intensity decrease is understood in terms of a lower carrier concentration in the thicker samples while the shift in energy may be due to interacting wells.

\section{References}

[1] K. Eberl, S.S. Iyer, S. Zoliner, J.C. Tsang, F.K. LeGoues, Appl. Phys. Lett. 60, 3033 (1992).

[2] T.P. Pearsall, J. Bevk, L.C. Feldman, J.M. Bonar, J.P. Mannaerts, A. Ourmazd, Phys. Rev. Lett. 58, 729 (1987).

[3] T. Pearsall, J.M. Vandenberg, R. Hull, J.M. Bonar, Phys. Rev. Lett. 63, 2104 (1989).

[4] R. Zachai, K. Eberl, G. Abstreiter, E. Kasper, H. Kibbel, Phys. Rev. Lett. 64, 1055 (1990). 
[5] J. Olajos, J. Engvall, H.G. Grimmeiss, U. Menczigar, G. Abstreiter, H. Kibbel, E. Kasper, H. Presting, Phys. Rev. B 46, 12857 (1992).

[6] U. Menczigar, G. Abstreiter, J. Olajos, H.G. Grimmeiss, Phys. Rev. B 47, 4099 (1993).

[7] U. Gnutzmann, K. Clausecker, Appl. Phys. 3, 9 (1974).

[8] R.J. Turton, M. Jaros, I. Morrison, Phys. Rev. B 38, 8397 (1988).

[9] M. Jaros, K.B. Wong, R.J. Turton, J. Electron. Maler. 19, 35 (1990).

[10] R.J. Turton, M. Jaros, Mater. Sci. Eng. B 7, 37 (1990).

[11] U. Schmid, N.E. Christensen, M. Cardona, M. Alouani, in: Proc. 20th ICPS, Eds. E.M. Anastassakis, J.D. Joannopoulos, World Scientific, Singapore 1990, p. 865.

[12] U. Schmid, F. Lukes, N.E. Christensen, M. Alouani, M. Cardona, E. Kasper, H. Kibbel, H. Presting, Phys. Rev. Lett. 65, 1933 (1990).

[13] C. Tserbak, H.M. Polatoglou, G. Theodorou, Phys. Rev. B 47, 7104 (1993).

[14] E. Kasper, Surf. Sci. 174, 630 (1986).

[15] J. Olajos, J. Engvall, H.G. Grimmeiss, H. Kibbel, E. Kasper, H. Presting, Thin Solid Films 222, 243 (1992).

[16] J. Olajos, Ying-Bo Yia, J. Engvall, H.G. Grimmeiss, H. Kibbel, H. Presting, E. Kasper, Phys. Rev. B 49, 2615 (1994).

[17] J. Engvall, J. Olajos, H.G. Grimmeiss, H. Presting, H. Kibbel, E. Kasper, Appl. Phys. Lett. 63, 491 (1993). 